\title{
Filosofía lean y gerencia de operaciones: El caso de las empresas de Ambato, Ecuador
}

\author{
Marisol Ortega, Homero Vaca \\ Departamento de Ciencias Económicas, Administrativas y del Comercio, Universidad de las Fuerzas Armadas ESPE, \\ Extensión Latacunga \\ ymortega@espe.edu.ec,shvaca@espe.edu.ec
}

\begin{abstract}
Resumen-El propósito de este estudio es brindar, desde la perspectiva de la filosofía Lean, una visión panorámica del manejo de los procesos en las empresas del Cantón Ambato, Ecuador, a través de la cual se determinan los aspectos claves de este sistema de trabajo con énfasis en los procedimientos para la eliminación de desperdicios que no aportan valor. La metodología se basa en un estudio macro del sector industrial y comercial, llevado a cabo en el 2016. La recopilación de información se realiza mediante un estudio de campo con entrevistas estructuradas, dirigidas a los administradores de las empresas. Los resultados obtenidos reflejan la cultura en el campo de la optimización de recursos, así como también demuestran el nivel de conocimientos del modelo especialmente en términos de desperdicios, sobreproducción, distribución del espacio y mejora continua. En las conclusiones se deduce que las entidades de la zona tienen implementados mecanismos de mejora continua en los procesos, aunque ciertas prácticas para cumplir con los propósitos Lean requieren ser perfeccionadas.
\end{abstract}

Palabras Claves-Ambato, desperdicios, empresa, filosofía Lean, mejora continua, operaciones.

Abstract-The purpose of this study is to offer, from the perspective of the Lean philosophy, a panoramic vision of the management of the processes in the companies of Canton Ambato, Ecuador, through which the key aspects of this work system are determined with emphasis in the procedures for the elimination of waste that does not add value. The methodology is based on a macro study of the industrial and commercial sector, carried out in 2016. The information is collected through a field study with structured interviews, aimed at company managers. The results obtained reflect the culture in the field of resource optimization, as well as demonstrate the level of knowledge of the model especially in terms of waste, overproduction, space distribution and continuous improvement. In the conclusions, it is deduced that the entities of the zone have implemented mechanisms of continuous improvement in the processes, although certain practices to fulfill the Lean purposes need to be perfected.

Keywords-Ambato, waste, company, Lean philosophy, continuous improvement, operations.

\section{INTRODUCCIÓN}

La actividad socio-productiva y comercial de la provincia de Tungurahua y concretamente del Cantón Ambato en la última década se ha caracterizado porque hay “[...] más producción, más ventas, más inversión en maquinaria e infraestructura, más crédito y más capacitación. Casi todos los sectores productivos se han revitalizado: el calzado y cuero, el carrocero, el textil, el comercio y la agricultura [1].

Con este preámbulo, en este artículo investigativo se enuncian desde un enfoque cualitativo los puntos de vista y tendencias referidos a la práctica de la filosofía Lean en las organizaciones industriales y de servicios de la zona de Ambato en el contexto de eliminación de desperdicios como una alternativa para optimizar el uso de recursos; además se recopila los fundamentos teóricos importantes con el propósito de esquematizar los temas de mejora continua. Las razones que motivaron la realización de este estudio subyacen en la necesidad de explorar las características innovadoras de trabajo en las empresas del cantón en materia de procesos, aspectos que se han evidenciado en su posicionamiento económico nacional e internacional, por lo que se incursiona en este ámbito a través de un sondeo programado. Con este criterio se aprovecha la oportunidad de conocer de cerca los mecanismos de gestión de operaciones en tales entidades, que, en determinado momento, pueden ser el modelo a seguir en otros sectores del emprendimiento, sentando así las bases para futuros trabajos de investigación.

Con éstos antecedentes, se explica la estructura de este documento, misma que inicia con la sección II, en la que se expone las bases teóricas de la investigación, así como también el método aplicado. Seguidamente, en la sección III se presenta el análisis de los resultados mediante tablas y figuras ilustrativas; en la sección IV, se formulan las conclusiones; y, en la parte final, se establecen las referencias, las cuales son plenamente sustentadas.

\section{MÉTODO}

\section{A. Filosofía Lean}

Etimológicamente "la palabra "lean" en inglés significa "magra”, es decir, sin grasa. En español no combina mucho la definición de "manufactura magra", por lo que se le ha llamado: Manufactura Esbelta o Manufactura Ágil, pero al igual que muchos otros términos en inglés, se prefiere dejarlo así”. (Padilla, 2010:65).

La filosofía Lean históricamente nace en Japón por los años 50 sentando las bases para la mejora continua, el cual es renovado desde una dimensión paradigmática en el año 90 enfocado a la metodología para eliminación de desperdicios por James P. Womack, Daniel T. Jones, Daniel Roos, en su libro titulado The Machine that changed the World [2]. (La máquina que cambió al mundo). En este contexto, "la nueva filosofía de producción ya era conocida en otras latitudes, de diferentes maneras, entre ellas "producción sin pérdidas", "nuevo sistema de producción" o "manufactura de clase mundial”, y fue implementada en otros campos como la 
administración y el desarrollo de productos” [3, p. 34]. Esta reflexión tiene mucho que ver con la implementación sostenible de los procesos, criterios de relación de largo plazo, respeto y beneficio mutuo, mejora continua [4, p. 121]

En conclusión, “el término Lean (Esbeltez) se enfoca en la reducción de desperdicio, de eliminar todo aquello que no necesita para manufacturar un producto o servicio y es manifestado en un énfasis al flujo” [6]

\section{B. Desperdicios}

El término desperdicio proviene del Japonés Muda, que significa despilfarro, “específicamente toda aquella actividad humana que absorbe recursos, pero no crea valor: fallos que precisan rectificación, producción de artículos que nadie desea y el consiguiente amontonamiento de existencias y productos sobrantes [7]

Continuando, "Hay ocho clases de muda: sobreproducción, desperdicio, transporte, procesamiento, inventario, movimiento, repeticiones, y utilización deficiente del personal. La meta es tratar de eliminar o reducir estos costos” [8,p 4]; costos por “ desperdicios, organización del área de trabajo, reducción del tiempo de preparación, reducción de los niveles de inventario” [6, p. 82].

\section{Gestión de operaciones}

En el ámbito empresarial, se lo definiría como "la estandarización de las operaciones en producción, es decir las hojas de operación estándar [...]. Las operaciones estándar se establecen para cada operación unitaria, por cada parte, por cada máquina y por cada proceso” [9, p. 85]. Las operaciones estándar son el mejor método para que se "aprendan correctamente las operaciones, hacer que la repitan cuantas veces sea necesario y si comete errores corregirlos inmediatamente. Sin falta verificar la calidad del producto terminado. [9, pp 104-108]; esta norma por consiguiente, es vista como: "Una filosofía de gestión que genera cambios o pequeñas mejoras incrementales en el método [...] (o procesos de trabajo) que permite reducir despilfarros $y$ por consecuencia mejorar el rendimiento del trabajo, llevando a la organización a un espiral de innovación incremental. . [10, p 285].

\section{Mejora continua}

Según la NTP-ISO 9000:2001, Mejora continua es una "actividad recurrente para aumentar la capacidad para cumplir los requisitos" siendo los requisitos la "necesidad o expectativa establecida, generalmente implícita u obligatoria". [11, p. 92]. Se relaciona con el "enfoque a los procesos, por que plantea el ciclo de mejora continua de los procesos PHVA que significa "Planificar-HacerVerificar-Actuar" desarrollado por W. Shewarth (1920) y conocido gracias a W. Edwards Deming [...] como el Ciclo DEMING. Ídem.

En este mismo ámbito se puede mencionar también a otro modelo de apoyo; este es el modelo Kaizen, "una herramienta de clase mundial que ayuda a las empresas a la mejora continua, cuyo objetivo es la calidad, la competitividad y la productividad. [12, p. 333].

\section{E. SMED}

Significa "Cambio de modelo en minutos de un sólo dígito”, técnica para reducir los tiempos de alistamiento de una actividad u operación con el propósito de mejorar las operaciones de cambio de modelo en menos de 10 minutos. Desde la última pieza buena hasta la primera pieza buena en menos de 10 minutos. El sistema SMED nació por necesidad para lograr la producción Justo a Tiempo. Este sistema fue desarrollado para acortar los tiempos de la preparación de máquinas, posibilitando hacer lotes más pequeños de tamaño. [13].

Esto significa que las actividades de producción o de distribución deben someterse a un proceso riguroso de control de tiempos, para evitar las filas de espera y los consecuentes retrasos en la entrega del producto final.

\section{F. Método}

La metodología es un estudio de investigación de campo enfocado a un grupo de empresas de la ciudad de Ambato. El método seleccionado es el muestreo probabilístico aleatorio simple, cuyo soporte de recopilación de datos es la "entrevista estructurada" con preguntas dicotómicas, dirigida a los administradores de los negocios. Los criterios para la aplicación de este método se respaldan en las características homogéneas de la población, aspecto que permite escoger con libertad un conjunto aleatorio de unidades de estudio que en este caso fueron 84 empresas, de las cuales 24 son industriales y 60 de servicios. El estudio lo ejecutó un equipo de trabajo conjuntamente con un coordinador. Finalmente, los datos recogidos fueron procesados en el sistema informático.

\section{ANÁLISIS DE RESUltAdOS}

La investigación de campo estuvo dirigida a las empresas, en función de su actividad propia, mismas que se detallan en las Tablas I y II:

TABLA I

LISTADO DE EMPRESAS INDUSTRIALES

No. NOMBRE DE LA EMPRESA

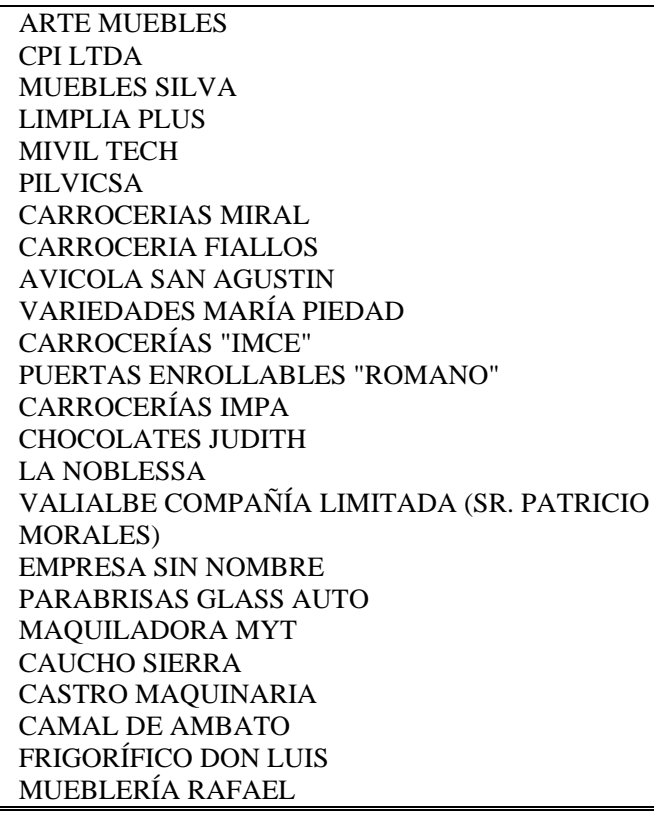


Para la fase de consulta in situ, se estructuró un formulario o cédula de entrevista con 15 ejes temáticos, de los cuales se seleccionaron nueve por considerarse los más relevantes. Las preguntas formuladas son dicotómicas con opciones de respuesta de SI o NO, que representan la afirmación del conocimiento o desconocimiento del entrevistado acerca de cada uno de los tópicos. Los resultados son presentados en términos porcentuales a continuación en la Tabla III y la Fig. 1.

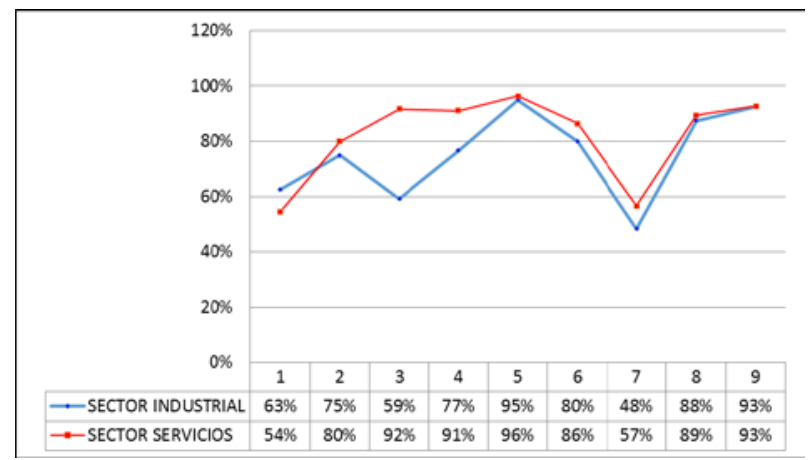

Fig. 1. Representación gráfica de la relación sobre las prácticas Lean en los dos sectores

\section{ANÁLISIS E INTERPRETACIÓN DE LAS PREGUNTAS}

Primeramente, el promedio general sobre prácticas Lean es 78,5\%. En el sector industrial, se verifica que existe conocimiento del sistema en un $63 \%$ contra 37 , que es el desconocimiento; en sector servicios el porcentaje afirmativo es $54 \%$ contra el $46 \%$, negativo; porcentajes que permiten establecer que el sistema Lean es aplicado en la mayor parte de empresas, las diferencias entre el uno y otro son justificables por cuanto el pensamiento Lean, históricamente ha sido más asociado a la manufactura antes que a la distribución o el comercio.

En segundo lugar, se puede comprender que el nivel máximo de eficiencia, entre las dimensiones Lean, le corresponde a la distribución por áreas de trabajo, que alcanza una cota del 95 y el 96\% respectivamente, aspecto positivo por cuanto las organizaciones en su mayoría prefieren trabajar en espacios bien distribuidos. Del lado contrario, están las cotas más bajas del 48 y $57 \%$, que es la aplicación de la técnica SMED, la cual revela que no se le presta mucha atención a los tiempos controlados.

\section{CONCLUSIONES}

La gestión de las empresas demuestra un alto nivel de responsabilidad en el control de desperdicios y prácticas de mejora continua. En los dos sectores existe gran apertura hacia los cambios para mejorar la calidad de sus productos o servicios. Se evidencia además, la similitud de ideologías y pensamientos en cuanto a la orientación al servicio, siendo por lo tanto una característica referente de desarrollo productivo. El análisis sobre los conocimientos del manejo controlado en los tiempos de procesos, refleja no estar en los niveles óptimos requeridos, aspecto que en cierto modo puede significar incremento de costos.
TABLA II

LISTADO DE EMPRESAS DE SERVICIOS

\begin{tabular}{|c|c|c|c|}
\hline NO & NOMBRE DE LA EMPRESA & $\begin{array}{c}\text { NO } \\
.\end{array}$ & $\begin{array}{l}\text { NOMBRE DE LA } \\
\text { EMPRESA }\end{array}$ \\
\hline 1 & $\begin{array}{l}\text { AUTOMOTORES DE LA } \\
\text { SIERRA }\end{array}$ & 31 & $\begin{array}{l}\text { ROTULOS (SIN } \\
\text { NOMBRE) }\end{array}$ \\
\hline 2 & HIDRORIEGO & 32 & OLAAUTO \\
\hline 3 & CAFETERÍA BUBBLE CREP & 33 & MODUPAL \\
\hline 4 & $\begin{array}{l}\text { LIBRERÍA D’LUISIN } \\
\text { LUISIN LIBRERÍA Y }\end{array}$ & 34 & WILSON SPORT \\
\hline 5 & $\begin{array}{l}\text { ACCESORIOS PARA } \\
\text { COMPUTADORA }\end{array}$ & 35 & EP-EMAPA-A \\
\hline 6 & AGRO AHORRO & 36 & EXREME WARE \\
\hline 7 & TRONICK & 37 & $\begin{array}{l}\text { ESTACION DE } \\
\text { SERVICIO SUR }\end{array}$ \\
\hline 8 & $\begin{array}{l}\text { PREUNIVERSITARIOWILLIAM } \\
\text { SIDIS }\end{array}$ & 38 & $\begin{array}{l}\text { ONG DANIELLE } \\
\text { CHILDRENS FUND- } \\
\text { ECUADOR }\end{array}$ \\
\hline 9 & PRODENTAL & 39 & $\begin{array}{l}\text { IMPORTADORA } \\
\text { MERCANTIL }\end{array}$ \\
\hline 10 & PAPELRECICLC. & 40 & EMPRESA 2: NARESA \\
\hline 11 & FARMACIA COMUNITARIA & 41 & $\begin{array}{l}\text { EMPRESA 3: CHINA } \\
\text { AUTO PARTS }\end{array}$ \\
\hline 12 & GLOBSEG & 42 & HINO \\
\hline 13 & SHULY - PORCELANICRON & 43 & SAMMY MASAJES \\
\hline 14 & ELECTRÓNICA MANTILLA & 44 & IP AMBATO \\
\hline 15 & $\begin{array}{l}\text { CONSTRUCCIONES SÁNCHEZ } \\
\text { E HIJOS }\end{array}$ & 45 & TROFEOS ESPINOZA \\
\hline 16 & $\begin{array}{l}\text { EMPRESA 3:IMPORTADORA } \\
\text { KUMHO }\end{array}$ & 46 & CHIC BROSTER \\
\hline 17 & HOSTERIA MANTELES & 47 & COORPA \\
\hline 18 & HOSTAL CASA DEL VALLE & 48 & AMBAGRO \\
\hline 19 & $\begin{array}{l}\text { APPOL SERVICES } \\
\text { CAPACITACIONES }\end{array}$ & 49 & $\begin{array}{l}\text { QUÍMICOS PARA } \\
\text { PRODUCTOS LÁCTEOS }\end{array}$ \\
\hline 20 & $\begin{array}{l}\text { INTERLAB LABORATORIO } \\
\text { CLINICO }\end{array}$ & 50 & $\begin{array}{l}\text { RESTAURANT A } \\
\text { FUEGO LENTO }\end{array}$ \\
\hline 21 & $\begin{array}{l}\text { ISAAC NEWTON } \\
\text { PREUNIVERSITARIO }\end{array}$ & 51 & $\begin{array}{l}\text { LABORATORIO } \\
\text { CLÍNICO CENTRAL }\end{array}$ \\
\hline 22 & $\begin{array}{l}\text { SERVICIOS ELÉCTRICOS } \\
\text { NAVCAS CIA. LTDA }\end{array}$ & 52 & MEMORY'S STUDIO \\
\hline $\begin{array}{l}23 \\
24\end{array}$ & $\begin{array}{l}\text { ESU CATERING } \\
\text { SIDEPRO S.A. }\end{array}$ & $\begin{array}{l}53 \\
54\end{array}$ & $\begin{array}{l}\text { VISPRIN CIA. LTDA } \\
\text { SETH S.A }\end{array}$ \\
\hline 25 & HELADOS TATUS & 55 & $\begin{array}{l}\text { RESTAURANTE } \\
\text { "MORENA" }\end{array}$ \\
\hline 26 & CHOCOLATES D' MANUEL & 56 & $\begin{array}{l}\text { RESTAURANTE } \\
\text { "DOBLE FILO" }\end{array}$ \\
\hline 27 & $\begin{array}{l}\text { ACATIP TRANSPORTE DE } \\
\text { PASAJEROS }\end{array}$ & 57 & CORPOAMBATO \\
\hline 28 & HOTEL SAHARA & 58 & $\begin{array}{l}\text { HOSTAL SAN PEDRITO } \\
\text { DE PELILELO }\end{array}$ \\
\hline 29 & AUTO TOTAL & 59 & $\begin{array}{l}\text { LAVANDERIA MAGIC'S } \\
\text { DE PELILEO }\end{array}$ \\
\hline 30 & $\begin{array}{l}\text { FRENOS Y EMBRAGUES } \\
\text { "PALCA" }\end{array}$ & 60 & $\begin{array}{l}\text { COOPERATIVA } 22 \text { DE } \\
\text { JULIO DE TRANSPORTE }\end{array}$ \\
\hline
\end{tabular}

TABLA III

PREGUNTAS Y RESPUESTAS DE LA ENTREVISTA.

\begin{tabular}{|c|c|c|c|c|c|}
\hline \multirow{2}{*}{$\begin{array}{l}\mathbf{N} \\
\mathbf{0}\end{array}$} & \multirow{2}{*}{ PREGUNTA } & \multicolumn{2}{|c|}{$\begin{array}{l}\text { INDUSTRI } \\
\text { A }\end{array}$} & \multicolumn{2}{|c|}{$\begin{array}{l}\text { SERVICIO } \\
\text { S }\end{array}$} \\
\hline & & $\overline{\text { SI }}$ & NO & SI & NO \\
\hline 1 & $\begin{array}{l}\text { ¿Sabe usted lo que es la filosofía } \\
\text { Lean? }\end{array}$ & $63 \%$ & $37 \%$ & $54 \%$ & $46 \%$ \\
\hline 2 & $\begin{array}{l}\text { ¿Conoce usted lo que significa la } \\
\text { reducción de desperdicios? }\end{array}$ & $75 \%$ & $25 \%$ & $80 \%$ & $20 \%$ \\
\hline 3 & $\begin{array}{l}\text { ¿Está usted de acuerdo con la } \\
\text { sobreproducción? }\end{array}$ & $59 \%$ & $41 \%$ & $92 \%$ & $8 \%$ \\
\hline 4 & $\begin{array}{l}\text { ¿Conoce usted el significado de } \\
\text { producción o distribución bajo } \\
\text { pedido? }\end{array}$ & $77 \%$ & $23 \%$ & $91 \%$ & $9 \%$ \\
\hline 5 & $\begin{array}{l}\text { ¿Conoce usted la importancia de la } \\
\text { distribución por áreas de trabajo? }\end{array}$ & $95 \%$ & $5 \%$ & $96 \%$ & $4 \%$ \\
\hline 6 & $\begin{array}{l}\text { ¿Usted ha aplicado la producción o } \\
\text { distribución Just in time? }\end{array}$ & $80 \%$ & $20 \%$ & $86 \%$ & $14 \%$ \\
\hline 7 & $\begin{array}{l}\text { ¿Ha aplicado alguna vez la técnica } \\
\text { SMED? }\end{array}$ & $48 \%$ & $52 \%$ & $57 \%$ & $43 \%$ \\
\hline 8 & $\begin{array}{l}\text { ¿Aplica usted métodos de prevención } \\
\text { de errores? }\end{array}$ & $88 \%$ & $12 \%$ & $89 \%$ & $11 \%$ \\
\hline 9 & $\begin{array}{l}\text { ¿Su empresa aplica permanentemente } \\
\text { la mejora continua? }\end{array}$ & $93 \%$ & $7 \%$ & $93 \%$ & $7 \%$ \\
\hline & "PROMEDIO: & $75 \%$ & $25 \%$ & $82 \%$ & $18 \%$ \\
\hline
\end{tabular}




\section{REFERENCIAS}

[1] W.-K. Chen, Linear Networks and Systems. Belmont, CA, USA: Wadsworth, 1993, pp. 123-135.

[2] El Comercio. "El ejemplo de Ambato y Tungurahua", El Comercio. [En línea]. Disponible en: http://www.elcomercio.com/opinion/ejemplo-ambato-tungurahuaopinion-produccion.html. [Accedido: 30-abr-2018].

[3] J. P. Womack, D. T. Jones, y D. Roos, "La máquina que cambió el mundo" La historia de la Produccion Lean, el arma secreta de Toyota que revolucióno la industria mundial del automóvil. 1era ed, Barcelona, España, Profit Editorial, 2017

[4] H. P. Díaz, O. G. S. Rivera, y J. A. G. Guerra, "Filosofía Lean Construction para la gestión de proyectos de construcción", vol. 11, n.o 1, pp. 32-53, 2014.

[5] A. Toledano de Diego, N. Mañes Sierra, y S. J. García, “ Las claves del éxito de Toyota”. LEAN, más que un conjunto de herramientas y técnicas, Cuad. Gest., vol. 9, n.o 2, 2009.

[6] L. Padilla, "Lean manufacturing manufactura esbelta/ágil", Rev. Electrónica Ing. Prim. ISSN, vol. 2076, p. 3166, 2010

[7] G. Maldonado Villalva, "Herramientas y técnicas lean manufacturing en sistemas de producción y calidad", 2008.

[8] D. T. Jones y J. P. Womack, Lean Thinking "Cómo utilizar el pensamiento Lean para eliminar los despilfarros y crear valor en la empresa." Grupo Planeta Spain, 2012.

[9] F. E. Meyers, "Diseño de instalaciones de manufactura y manejo de materiales." Pearson Educación, 2006

[10] F. González Correa, "Manufactura Esbelta (Lean Manufacturing). Principales Herramientas", RAITES Antes Panor. Adm., vol. 1, n.o 2, pp. 85-112, 2007

[11] M. F. S. Barraza y J. Á. M. Dávila, "Encontrando al Kaizen: Un análisis teórico de la Mejora Continua", Pecvnia Rev. Fac. Cienc. Económicas Empres. Univ. Ón, n.o 7, pp. 285-311, 2008.

[12] M. García, C. Quispe, y L. Ráez, "Mejora continua de la calidad en los procesos", Ind. Data, vol. 6, n.o 1, 2003

[13] A. G. Elizondo, "Kaizen, una mejora continua", Cienc. UANL, vol. 8, n.o 3, p. 330, 2005

[14] E. Infante Díaz, E. delaCruz, y D. Alexander, "Propuesta de mejoramiento de la productividad de la línea de camisetas interiores en una empresa de confecciones por medio de la aplicación de herramientas lean manufacturing", Bibl. USB Cali T6585 I43p CD-ROM, 2013 